\title{
Progesterone effects on mouse sperm kinetics in conditions of viscosity
}

\author{
S Pérez-Cerezales ${ }^{1}$, A P López-Cardona ${ }^{1,2}$ and A Gutiérrez-Adán ${ }^{1}$ \\ ${ }^{1}$ Departamento de Reproducción Animal, INIA, Avda. Puerta de Hierro, Madrid, Spain and ${ }^{2}$ Grupo de Investigación \\ en Biogénesis, Universidad de Antioquia, Antioquia, Colombia
}

Correspondence should be addressed to S Pérez-Cerezales; Email: perez.serafin@inia.es

\begin{abstract}
The spermatozoa delivered to the female genital tract need to swim towards the oocyte through viscous secretions. Once close to the oocyte, the spermatozoa are guided by a gradient of progesterone $\left(P_{4}\right)$ and other unknown chemoattractants via a process known as chemotaxis. Using polyvinylpyrrolidone to establish the conditions of viscosity, we examined the response of mouse spermatozoa to $\mathbf{P}_{4}$. Herein, we show that in low-viscous media, $\mathbf{P}_{4}$ induces hyperactive-like motility whereby sperm show erratic trajectories and non-progressive movement. However, an opposite response is produced in viscous medium in that trajectories are linear and motility is more progressive and less erratic. Our observations provide a behavioural explanation for the chemotaxis of spermatozoa swimming under viscous conditions in a spatial gradient of the chemoattractant $\mathbf{P}_{4}$. They also highlight the importance of using viscous solutions to mimic in vivo conditions when analysing sperm behaviour in response to any stimulus.

Reproduction (2016) 151 501-507
\end{abstract}

\section{Introduction}

Once in the female genital tract, to reach the fertilization site, spermatozoa need to tackle several anatomical structures, each with specific and complex physicochemical properties (Suarez \& Pacey 2006). Active mechanisms will guide the spermatozoa to the proximity of the oocyte (Eisenbach \& Giojalas 2006). So far, three such guidance mechanisms have been proposed: temperature gradient (thermotaxis), against fluid flow (rheotaxis) and chemoattractant(s) gradient (chemotaxis). All the three mechanisms are functional in the oviduct (see Pérez-Cerezales et al. (2015) for a review).

A feature shared by the three guidance mechanisms is that spermatozoa show a hyperactivation-like motility (Armon \& Eisenbach 2011, Miki \& Clapham 2013, Boryshpolets et al. 2015). Hyperactivation is a property of capacitated spermatozoa that, under low-viscous conditions, involves vigorous movements produced by asymmetrical and high-amplitude waves in the flagella, resulting in erratic swimming trajectories (Suarez 2008). Currently, the model suggested for spermatozoa swimming in a spatial gradient of temperature (Boryshpolets et al. 2015) or chemoattractant (Gakamsky et al. 2009, Armon \& Eisenbach 2011) is similar to that described for bacterial chemotaxis (Macnab \& Koshland 1972). According to this model, a spermatozoon swimming in a gradient towards a lower temperature or lower chemoattractant concentration responds by increasing the frequency of hyperactivation-like events and turns to modify its direction of swimming. Conversely, when swimming towards a higher temperature or chemoattractant concentration, this constant stimulation causes the suppression of turns and hyperactive-like events. If no changes are perceived, the spermatozoon recovers its non-stimulated swimming mode, consisting of a straighter movement pattern with occasional hyperactivation events and turns. Although the model has been empirically validated for chemotaxis and thermotaxis, the behavioural response of spermatozoa to progesterone $\left(\mathrm{P}_{4}\right)$ as the main known chemoattractant has shown some discrepancies with the model. In studies addressing the response of a population of human spermatozoa to $\mathrm{P}_{4}(1-10 \mathrm{nM})$, enhanced hyperactivation-like motility events have been observed (Uhler et al. 1992, Gakamsky et al. 2009). This argues against the proposed model as increasing concentrations of chemoattractant should have a damping effect on hyperactivation-like motility. In an elegant series of experiments, Armon et al. (2011) showed that hyperactive-like motility of human spermatozoa was effectively reduced in the sperm subpopulation swimming towards higher concentrations of $\mathrm{P}_{4}$. However, the physiological meaning of $\mathrm{P}_{4}$ 's main effect of increasing the hyperactive-like motility of the general sperm population remains unknown.

It has been established that CatSper mediates $\mathrm{P}_{4}$-induced Ca ${ }^{2+}$ influx (Lishko et al. 2011, Strünker et al. 2011) as well as hyperactivation (Carlson et al. 2003) in human spermatozoa. However, studies conducted so far have failed to detect CatSper activation by $\mathrm{P}_{4}$ in mouse spermatozoa (Lishko et al. 2011), neither $P_{4}$-meditated chemotaxis (Chang et al. 2013). It has been shown in 
mice that the initial $\mathrm{Ca}^{2+}$ influx provoked by $\mathrm{P}_{4}$ occurs at the base of the sperm head (Fukami et al. 2003), whereas CatSper activation initiates $\mathrm{Ca}^{2+}$ influx in the main stretch of the flagellum (Xia et al. 2007). Moreover, $\mathrm{P}_{4}$ elicits transient $\mathrm{Ca}^{2+}$ influx in spermatozoa from Catsper1-null mice (Ren et al. 2001), suggesting that $\mathrm{P}_{4}$ might activate another $\mathrm{Ca}^{2+}$ influx pathway besides CatSper in mammalian sperm. It has been reported that $\mathrm{P}_{4}$ fails to induce $\mathrm{Ca}^{2+}$ fluctuations in $P / c \delta 4$-null mice (Fukami et al. 2003), suggesting PLC pathway involvement in this response, possibly via $\mathrm{Ca}^{2+}$ mobilization from internal $\mathrm{Ca}^{2+}$ stores at the base of the sperm head through the IP3 receptor (Darszon et al. 2011). Thus, given the localization of the $\mathrm{P}_{4}$ membrane receptor in mouse spermatozoa (Pietrobon et al. 2003), its activation by $\mathrm{P}_{4}$ might be the mechanism mediating the hyperactivation response, perhaps through the PLC pathway.

Interestingly, it has been noted that the behaviour of human spermatozoa in response to temperature changes varies under conditions of viscosity or low viscosity. Under viscous conditions considered closer to those of the physiological environment, the temperature-dependent hyperactive-like behaviour observed in low-viscous conditions changes to one showing a higher frequency of turns when the temperature is reduced (Boryshpolets et al. 2015). This indicates the different behaviour of spermatozoa in response to a stimulus depending on the viscosity of the surrounding medium. Indeed, hyperactivation-like motility in lowviscous conditions has been proposed to be the manifestation of a more efficient motility mode for sperm propagation under viscous conditions in hamster (Suarez et al. 1991) and mouse spermatozoa (Suarez \& Dai 1992). Thus, the hyperactive-like motility shown by spermatozoa swimming in low-viscous conditions in response to $\mathrm{P}_{4}$ could also indicate a more efficient swimming mode under physiological conditions of viscosity.

This study was designed to address the inconsistencies observed in the $\mathrm{P}_{4}$ chemotaxis model by determining the behaviour of mouse spermatozoa in response to this chemoattractant under conditions simulating the viscosity of the physiological environment of the female genital tract.

\section{Materials and methods \\ Reagents}

All media components were purchased from Sigma-Aldrich, except where otherwise stated.

\section{Spermatozoa}

Experiments with mice were carried out in strict accordance with recommendations of the guidelines of European Community Council Directive 86/609/EEC. Every effort was made to minimize suffering. Experiments were approved by the Committee on the Ethics of Animal Experiments of the INIA (permit number CEEA 2014/025).

For sperm analysis, F1 generation males from crosses between C57BL/6J (B6) female $\times \mathrm{DBA} / 2$ J (D2) male mice were used. The animals were kept in conditions of a $14 \mathrm{~h}$ light:10 h darkness cycle with no food or water restrictions. At the age of 4 months, males were individually isolated for 2 weeks before killing by cervical dislocation. Spermatozoa were collected from the caudal epididymis and suspended in human tubal fluid (HTF) medium $\left(2.04 \mathrm{mM} \mathrm{CaCl} \times 2 \mathrm{H}_{2} \mathrm{O}\right.$, $101.6 \mathrm{mM} \mathrm{NaCl}, 4.69 \mathrm{mM} \mathrm{KCl}, 0.37 \mathrm{mM} \mathrm{KH} \mathrm{PO}_{4}, 0.2 \mathrm{mM}$ $\mathrm{MgSO}_{4} \times 7 \mathrm{H}_{2} \mathrm{O}, 21.4 \mathrm{mM}$ sodium lactate, $0.33 \mathrm{mM}$ sodium pyruvate, $2.78 \mathrm{mM}$ glucose, $25 \mathrm{mM} \mathrm{NaHCO}, 100 \mathrm{U} / \mathrm{mL}$ penicillin, $50 \mu \mathrm{g} / \mathrm{mL}$ streptomycin $\mathrm{SO}_{4}$ and $0.001 \%(\mathrm{w} / \mathrm{v})$ phenol red, supplemented with $1 \%$ BSA and stabilized overnight at $37^{\circ} \mathrm{C}$ in a $5 \% \mathrm{CO}_{2}$ atmosphere). Once collected, the sperm samples were incubated for $1 \mathrm{~h}$ under an atmosphere of $5 \%$ $\mathrm{CO}_{2}$ at $37^{\circ} \mathrm{C}$ for capacitation.

\section{Sperm processing and motility}

After capacitation, spermatozoa were diluted to a final concentration of $\sim 2 \times 10^{6} / \mathrm{mL}$ in HTF with or without polyvinylpyrrolidone (PVP) of average MW360000 at 4, 6 or $7.5 \%$ $(\mathrm{w} / \mathrm{v})$. For each experiment with $\mathrm{P}_{4}, 20 \mu \mathrm{L}$ of $\mathrm{P}_{4}$ or DMSO (control) pre-diluted in HTF $(1: 10)$ were added to $100 \mu \mathrm{L}$ of spermatozoa diluted in HTF with or without PVP. Motility was then determined within $20 \mathrm{~s}$ of adding $\mathrm{P}_{4}$ or DMSO by placing $20 \mu \mathrm{L}$ of sperm suspension onto a pre-warmed slide on the stage heated to $37^{\circ} \mathrm{C}$ of a Nikon Eclipse 50i microscope (Nikon) fitted with a digital camera Basler A312f (Basler AG, Ahrensburg, Germany) capable of recording 25 frames/s. In each repetition, five videos of 1s (20-60 moving spermatozoa) were recorded in different fields on the top of the drop and analysed using the Integrated Semen Analysis System (ISAS; Projectes i ServeisR+D S.L., Valencia, Spain). In each experiment, three replicates for each condition/treatment were set up. The parameters analysed were as described by Mortimer et al. (2000): straight-line velocity (VSL; time-averaged velocity of the sperm head along a straight line from its first position to its last position, expressed in $\mu \mathrm{m} / \mathrm{s})$; curvilinear velocity $(\mathrm{VCL}$; time-averaged velocity of the sperm head along its actual curvilinear path, expressed in $\mu \mathrm{m} / \mathrm{s}$ ); average path velocity (VAP; velocity over an average path generated by a roaming average between frames, expressed in $\mu \mathrm{m} / \mathrm{s}$ ); linearity (LIN) (defined as $(\mathrm{VSL} / \mathrm{VCL}) \times 100$ ); straightness (STR) (defined as (VSL/ VAP) $\times 100$ ); wobble $($ WOB) (defined as $($ VAP/VCL $) \times 100)$; amplitude of lateral head (ALH) displacement (width of the lateral movement of the sperm head, expressed in $\mu \mathrm{m}$ ) and beatcross frequency (BCF; number of times the sperm head crosses the direction of movement per second, expressed in $\mathrm{Hz}$ ).

\section{Acrosome reaction}

The method used was based on acrosome staining using Pisum sativum lectin conjugated with FITC (PSA-FITC) following a standard protocol as described previously (Lybaert et al. 2009) with minor modifications. Briefly, $P_{4}$ at $0.015 \mathrm{pM}$, 
$15 \mathrm{pM}, 15 \mathrm{nM}, 15 \mu \mathrm{M}$ or the same volume of DMSO (as a negative control) was added to capacitated spermatozoa and these were immediately processed for acrosome staining. In parallel, spermatozoa were diluted in HTF with or without 6\% PVP (w/v) and also immediately processed for acrosome staining. As a positive control, spermatozoa were incubated for 30 min with $10 \mu \mathrm{M}$ of the ionophore A23187 to induce the acrosome reaction. The spermatozoa were washed twice in PBS by centrifugation (1 $\mathrm{min}$ at $500 \mathrm{~g}$ ) and subsequently smeared on a microscope glass slide and air-dried for $15 \mathrm{~min}$. Next, the slides were immersed in absolute methanol for $30 \mathrm{~s}$, air-dried and rinsed in PBS twice for 5 min before incubation with PSA-FITC (15 $\mu \mathrm{g} / \mathrm{mL}$ in PBS) in a humid box for $30 \mathrm{~min}$. Finally, the slides were washed with distilled water for $15 \mathrm{~min}$ and mounted with an aqueous mounting medium (Fluoromount G; EMS, Hatfield, PA, USA). Slides were examined in a fluorescence microscope and numbers of acrosome-reacted and non-acrosome-reacted spermatozoa were counted by randomly moving across different fields of the slide (counting 100 cells per slide). For each treatment, experiments were run in triplicate using two sperm samples from two different animals.

\section{Statistical analysis}

All statistical tests were performed using the software package SPSS Statistics 22.0 for Windows (IBM, Armonk, NY, USA). Results are provided as means \pm S.E.M. Means were compared and analysed by three-way ANOVA followed by Tukey's post hoc test. Significance was set at $P<0.05$.

\section{Results}

To establish the swimming pattern of mouse spermatozoa in viscous conditions, we examined sperm kinetics at four concentrations of PVP (0 (low-viscous medium), 4,6 and $7.5 \%$ (viscous media)). Differences in most of the variables recorded were detected between the viscous media and low-viscous medium. Velocities (VCL, VSL and VAP) decreased with increasing percentages of PVP in the medium $(P<0.05$, three-way ANOVA; Fig. 1A). The velocity ratios LIN and STR were only affected at the highest PVP concentration (7.5\%; $P<0.05$, three-way ANOVA; Fig. 1B), indicating equally proportional reductions in the three velocities when spermatozoa swam in a medium containing 4 and 6\% PVP. The other velocity ratio analysed, WOB, did not vary with PVP concentration. In addition, viscosity effects were also observed on motility patterns such that in the viscous media, the lateral displacement of the cell was reduced in an inverse manner with respect to the PVP concentration, reflected as a reduction in BCF and ALH with increasing PVP percentage $(P<0.05$, three-way ANOVA; Fig. 1C). Accordingly, PVP led to the reduced velocity of spermatozoa and reduced side-to-side movement of the head and frequency of this movement, leading to a slower, more regular swimming trajectory (Fig. 1D). In the absence of
PVP, the swimming pattern was erratic, including more irregular side-to-side head movements.

We then went on to assess the dose-dependent effect of $\mathrm{P}_{4}$ on spermatozoa in low-viscous vs viscous medium. For these experiments, 6\% PVP was selected as the highest concentration for which relationships among the three velocities defined were unaffected, determining that STR, WOB and LIN remained unchanged. This means that 6\% PVP does not affect the STR of the sperm swimming trajectory and that any change in sperm behaviour could be attributed to their response to $P_{4}$. Moreover, this percentage of PVP did not induce the acrosome reaction (Fig. 2), which could in itself affect the behaviour of the spermatozoa.

In low-viscous medium, $\mathrm{P}_{4}$ reduced the LIN of sperm movement indicated by reduced values of LIN, STR and WOB $(P<0.05$, three-way ANOVA; Fig. 3). These effects were already observed at the lowest $P_{4}$ concentration tested $(P<0.05$, three-way ANOVA). Such changes produced in LIN, STR and WOB (Mortimer 2000) reflect the effect of $P_{4}$ on sperm, provoking a slight increase in VCL and slight decreases in VSL and VAP (these effects alone were insignificant; three-way ANOVA; Fig. 3). In addition, $15 \mathrm{nM}$ and $15 \mu \mathrm{M}$ concentrations of $\mathrm{P}_{4}$ led to a greater $\mathrm{ALH}(P<0.05$, three-way ANOVA), whereas no effects were observed on BCF. These observations indicate that the vigorous motility induced by $\mathrm{P}_{4}$ consisted of a greater sperm head

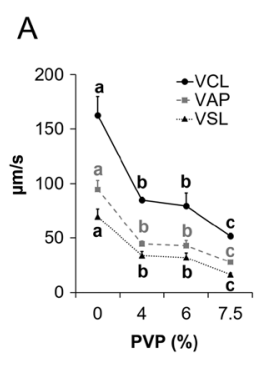

B
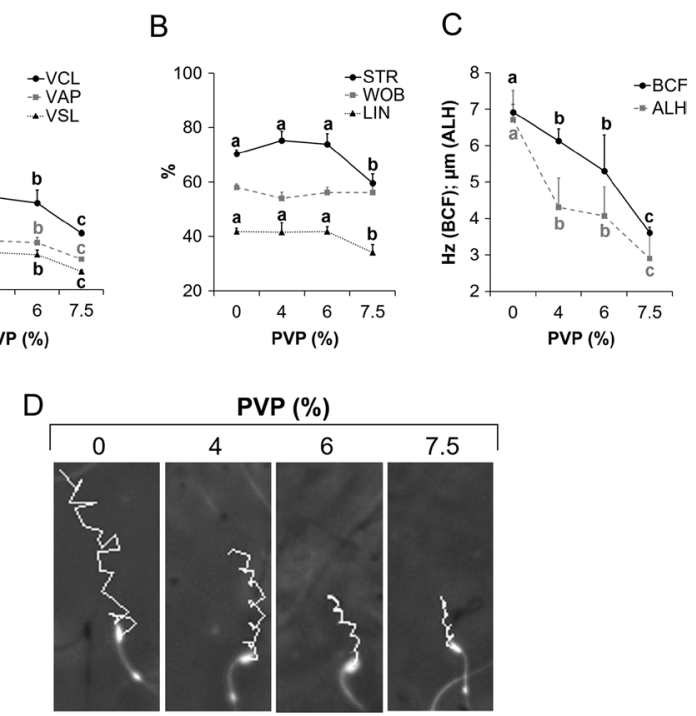

Figure 1 Sperm kinetics in viscous media. (A) Velocities: curvilinear (VCL), straight line (VSL) and average path (VAP). (B) Velocity ratios: linearity (LIN), straightness (STR) and wobble (WOB). (C) Head movements: beat-cross frequency (BCF, expressed in $\mathrm{Hz}$ ) and amplitude of lateral head displacement (ALH, expressed in $\mu \mathrm{m})$. (A, B and C) Different letters indicate significant differences between percentages of PVP according to three-way ANOVA $(P<0.05)$.

Mean \pm S.E.M., $n=5$ determinations; each determination is the average of measurements in five fields on 21-34 spermatozoa per field. (D) Sperm trajectories tracked in $1 \mathrm{~s}$ in the presence of different concentrations of PVP (from left to right: 0, 4, 6 and 7.5\%). 


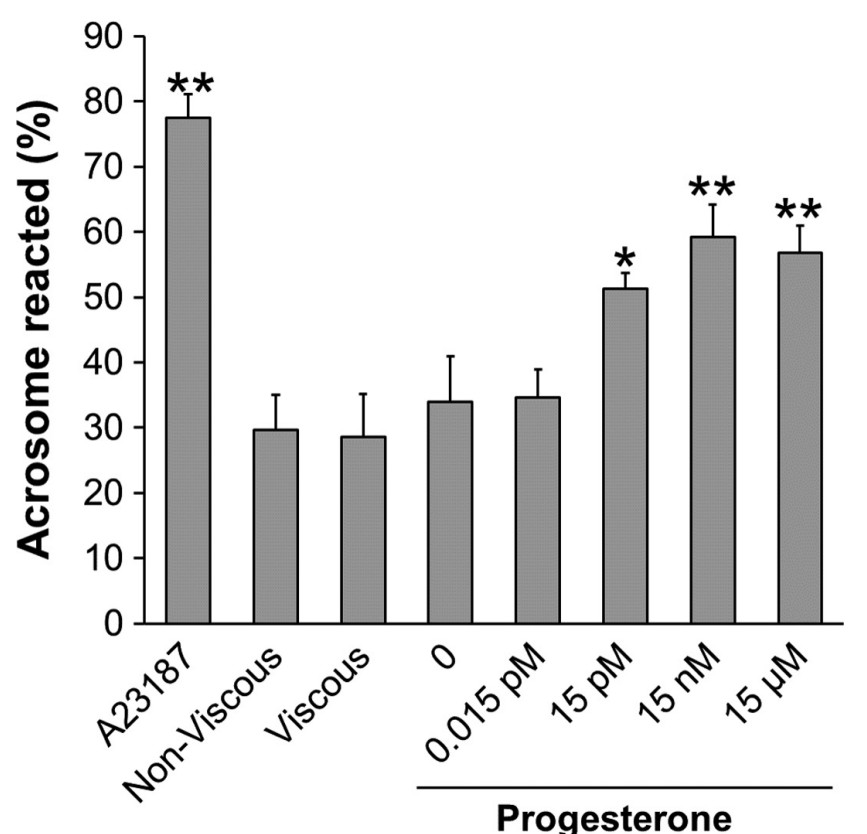

Figure 2 Acrosome-reacted spermatozoa visualized with Pisum sativum agglutinin conjugated to FITC (PSA-FITC) under different conditions. A23187 was used as a positive control to induce the acrosome reaction. $6 \%$ PVP was used for the viscosity conditions. As a negative control for progesterone $\left(\mathrm{P}_{4}\right.$; concentration 0$)$, DMSO (solvent) was added instead of $\mathrm{P}_{4}$ at the same volume. Varying concentrations of $\mathrm{P}_{4}$ were used to induce the acrosome reaction (0.015 pM, $15 \mathrm{pM}, 15 \mathrm{nM}$ and $15 \mu \mathrm{M}) .{ }^{*} P<0.05$ and ${ }^{* *} P<0.02$ according to three-way ANOVA. Mean \pm S.E.M., $n=8$ determinations; each determination is the average of measurements in 52-60 spermatozoa.

displacement distance but did not affect the frequency at which the head crossed the swimming trajectory. These changes in sperm kinetics are characteristic of a hyperactive-like motility, as described by others for human spermatozoa in response to $\mathrm{P}_{4}$ (Uhler et al. 1992, Gakamsky et al. 2009). $\mathrm{P}_{4}$ at $15 \mathrm{pM}, 15 \mathrm{nM}$ and $15 \mu \mathrm{M}$ induced the acrosome reaction (Fig. $2 ; P<0.05$, three-way ANOVA), meaning that the motility changes induced by $\mathrm{P}_{4}$ were accompanied by the release of acrosome contents.

In the viscous medium (6\% PVP), contrary effects of $\mathrm{P}_{4}$ to those produced in the low-viscous medium were observed. The $\mathrm{P}_{4}$ dose-dependent curves between low viscosity vs viscosity were significantly different for VSL, VAP, LIN, STR, WOB and ALH $(P<0.01$, three-way ANOVA; Fig. 3). Thus, sperm velocities (VSL and VAP) were increased (in response to $15 \mathrm{nM}$ and $15 \mu \mathrm{M} \mathrm{P}_{4}$; $P<0.05$, three-way ANOVA) and movement became linear as indicated by LIN and WOB ratios with increasing $\mathrm{P}_{4}$ concentrations (significant for $15 \mathrm{nM}$ and $15 \mu \mathrm{M}$ at $P<0.05$, three-way ANOVA) (Fig. 3). Moreover, $P_{4}$ led to a higher BCF (significant for $15 \mu \mathrm{M} ; P<0.05$, three-way ANOVA), whereas ALH was unaltered. These results suggest that the $\mathrm{P}_{4}$-induced non-progressive sperm motility (hyperactivation-like) observed in low-viscous medium is equivalent to the progressive motility observed under the more physiological conditions of the viscous media.

\section{Discussion}

Once in the female genital tract, spermatozoa have to swim in different mucous secretions whose viscosity differs according to anatomical site (Jansen 1978, Smith et al. 2009) and oestrous cycle stage (Jansen 1978, Miki \& Clapham 2013). Thus, the averaged viscosity of mouse uterus and oviduct is 81 and $1.8 \mathrm{cP}$ respectively (Miki \& Clapham 2013), in the range of viscosity obtained with the percentages of PVP (Hyakutake et al. 2015) used in our study. The effects of viscosity on mouse sperm kinetics observed here including a reduced velocity but more regular trajectory are in agreement with observations in hamster (Suarez et al. 1991), mice (Suarez \& Dai 1992) and cattle (Hyakutake et al. 2015). The different behaviour shown by spermatozoa in low-viscous compared with viscous media (Fig. 4A) suggests a need to study those factors determining the sperm motility mode under the latter conditions. $\mathrm{P}_{4}$ is among the most studied modulators of sperm function (Baldi et al. 2009). This hormone is secreted by cumulus cells and is a physiological sperm chemoattractant (Teves et al. 2006, Oren-Benaroya et al. 2008). In agreement with earlier data for human spermatozoa (Uhler et al. 1992, Gakamsky et al. 2009), under low-viscous conditions, we also observed hyperactivation-like motility in mouse spermatozoa in response to acute $\mathrm{P}_{4}$ treatment. Thus, $\mathrm{P}_{4}$ reduced LIN, STR and WOB and increased lateral displacement of the head (ALH; Figs 3 and $4 \mathrm{~A})$. As a consequence of these motility changes, the $\mathrm{P}_{4}$-activated spermatozoa acquired a more erratic swimming pattern, moving from side to side with higher amplitude (Fig. 4A). This motility type is known to facilitate spermatozoon progression under viscous conditions (Suarez et al. 1991, Suarez \& Dai 1992). Remarkably, under viscous conditions, we observed the opposite effects of $\mathrm{P}_{4}$ on sperm kinetics (Figs 3 and $4 \mathrm{~A}$ ). Thus, sperm LIN and BCF increased, whereas head displacement was reduced (Figs 3 and 4A). These results indicate that, contrary to what occurs in low-viscous media, the motility type induced by $\mathrm{P}_{4}$ is one of the propagation under viscous conditions. Therefore, it seems logical that the known non-progressive motility mode induced by $\mathrm{P}_{4}$ is actually an artefact of analysing the response under low-viscous conditions. This means that spermatozoa behave as expected for the model suggested for sperm chemotaxis when swimming in a spatial gradient of the chemoattractant $P_{4}$ (Armon \& Eisenbach 2011). Thereby, when a spermatozoon swims towards a higher concentration of $\mathrm{P}_{4}$, its motility becomes linearized, propagating the cell towards the higher concentrations (Fig. 4B). In contrast, a spermatozoon swimming 

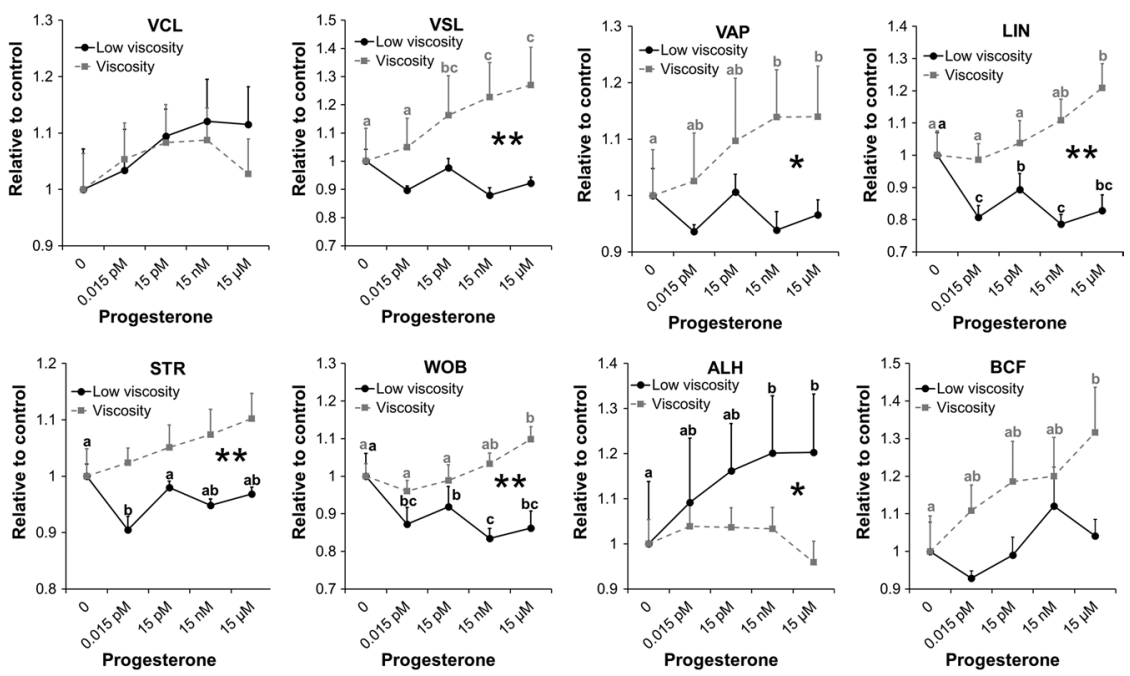

Figure 3 Sperm kinetics in response to progesterone $\left(\mathrm{P}_{4}\right)$ in low-viscous vs viscous medium. Different letters indicate significant differences between $\mathrm{P}_{4}$ concentrations in each viscosity condition according to three-way ANOVA $(P<0.05)$. Asterisks indicate significant differences between the slopes of the kinetic parameters for low viscosity vs viscosity according to three-way ANOVA $\left({ }^{*} P<0.05\right.$ and $\left.{ }^{* *} P<0.001\right)$. Mean \pm S.E.M., viscous medium ( $n=10$ determinations); low-viscous medium ( $n=7$ determinations). Each determination is the average of measurements in five fields (20-41 spermatozoa per field).

towards lower concentrations of $\mathrm{P}_{4}$ would show a more circular trajectory until higher concentrations of $\mathrm{P}_{4}$ linearized its motility again.

Chang et al. (2007) did not succeeded in detecting spatial chemotaxis in response to gradient of $\mathrm{P}_{4}$ in mouse spermatozoa when using conditions of low viscosity. Our results provide an explanation to this observation, as only under viscous conditions, the spermatozoa would migrate towards a $\mathrm{P}_{4}$ gradient in a chemotaxis spatial assay. Accordingly, the chemotaxis of human spermatozoa to $\mathrm{P}_{4}$ was reported using a chemotaxis spatial assay under viscous conditions (3.5\% PVP 25 K; Armon \& Eisenbach 2011). This demonstrates the importance of using conditions closer to physiological when studying the behavioural response of spermatozoa to any kind of stimuli. Complementary studies would include analyses of the response to $\mathrm{P}_{4}$ under other rheological conditions of the surrounding medium such as the viscoelasticity that characterize some fluids present along the female genital tract (Suarez \& Dai 1992). It has been reported that at similar viscosity, the VSL and flagellar beat frequency of cattle spermatozoa are higher in non-Newtonian fluids leading to a more linear motility type (Hyakutake et al. 2015) compared with Newtonian fluids. Accordingly, it has been shown that under viscoelastic conditions, hyperactive samples of mouse spermatozoa linearize the motility, whereas non-hyperactive spermatozoa behave in an opposite way (Suarez \& Dai 1992).

Our results also show that at a certain concentration (higher than $15 \mathrm{pM}$ ), $\mathrm{P}_{4}$ induces the acrosome reaction (Fig. 2). This observation is consistent with the dual effect of $\mathrm{P}_{4}$ on human spermatozoa, whereby it acts as a chemoattractant at lower concentrations and induces the acrosome reaction at higher concentrations (Uhler et al. 1992). This release of acrosome contents could be related to the $P_{4}$ gradient existing within the cumulus cells (Teves et al. 2006). Thus, we propose that $P_{4}$ could linearize motility when sperm swimming up the $\mathrm{P}_{4}$ gradient penetrate the cumulus and this could simultaneously provoke the release of acrosome contents to facilitate this penetration (Fig. 4B). This dual effect of $\mathrm{P}_{4}$ could only apply to certain sperm populations, as recently described in human spermatozoa (Uñates et al. 2014). Accordingly, the acrosome-reacting population would eliminate the barrier formed by the cumulus cells and the chemotactic population would progress towards the oocyte by acquiring a more linear path in response to the $\mathrm{P}_{4}$ gradient. It is also possible that both acrosome-reacting and chemotactic populations are comprised in part by the same spermatozoa. Indeed, it seems that most fertilizing spermatozoa undergo the acrosome reaction before reaching the zona pellucida of cumulus-enclosed oocytes (Jin et al. 2011). This would be an interesting case of altruism between 'sister' cells that needs to be further explored to extend the concept of cooperation between spermatozoa of a single ejaculate. Despite no descriptions to date in the laboratory mouse, heterogeneity among different sperm populations of the same ejaculate and cooperation phenomena have been observed in the wood mouse Apodemussylvaticus (Moore et al. 2002) and species of Peromyscus (Fisher \& Hoekstra 2010).

In conclusion, our findings suggest that the hyperactive-like motility induced by $\mathrm{P}_{4}$ in mouse spermatozoa swimming in low-viscous media is a manifestation of the chemotactic behaviour of spermatozoa that propagates them towards a chemoattractant gradient in viscous conditions. In view of the dramatically different responses to $\mathrm{P}_{4}$ produced in spermatozoa swimming in 

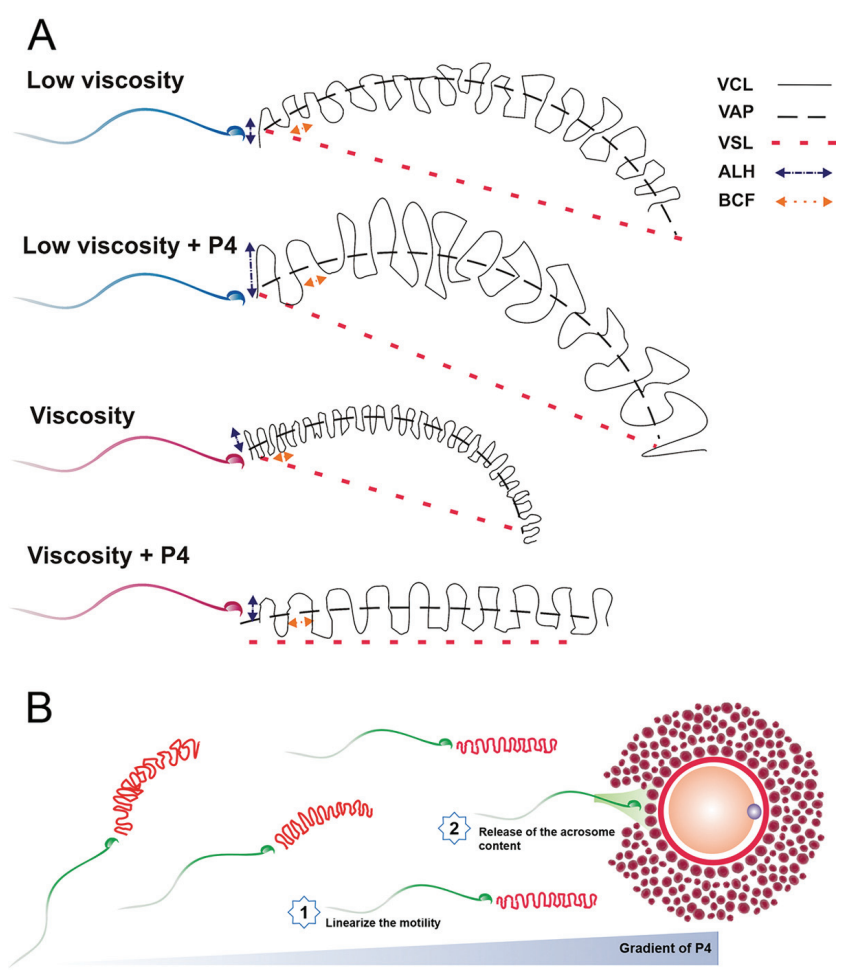

Figure 4 Models of the behavioural response of mouse spermatozoa to progesterone $\left(\mathrm{P}_{4}\right)$. (A) Trajectories of mouse spermatozoa swimming in viscous or low-viscous medium with or without $\mathrm{P}_{4}$. In low-viscous medium, the trajectory is more irregular than in viscous medium and $\mathrm{P}_{4}$ induces erratic motility. Under viscosity conditions, the trajectory is more regular and the addition of $\mathrm{P}_{4}$ linearizes motility facilitating sperm progression. (B) Dual effect of $\mathrm{P}_{4}$ on spermatozoa swimming in viscous medium towards the oocyte. (1) The trajectory becomes linear when spermatozoa swim towards higher $\mathrm{P}_{4}$ concentrations and is therefore guided by chemotaxis to the oocyte. (2) Once the spermatozoon reaches the granulosa, the higher concentration of $\mathrm{P}_{4}$ induces the acrosome reaction, facilitating penetration of the oocyte.

low-viscous or viscous media, we propose the viscosity of the medium is an essential factor to consider in any study designed to address sperm behaviour.

\section{Declaration of interest}

The authors declare that there is no conflict of interest that could be perceived as prejudicing the impartiality of the research reported.

\section{Funding}

This work was funded by Grant AGL2012-39652-C02-01 awarded by the Spanish Ministry of Economy and Competitiveness. S Pérez-Cerezales was supported by a postdoctoral grant from the same ministry. A P López-Cardona was supported by COLCIENCIAS (Francisco José de Caldas fellowship $512 / 2010)$.

\section{References}

Armon L \& Eisenbach M 2011 Behavioral mechanism during human sperm chemotaxis: involvement of hyperactivation. PloS ONE 6 e28359. (doi:10.1089/scd.2015.0322)

Baldi E, Luconi M, Muratori M, Marchiani S, Tamburrino L \& Forti G 2009 Nongenomic activation of spermatozoa by steroid hormones: facts and fictions. Molecular and Cellular Endocrinology 308 39-46. (doi:10.2741/3793)

Boryshpolets S, Pérez-Cerezales S \& Eisenbach M 2015 Behavioral mechanism of human sperm in thermotaxis: a role for hyperactivation. Human Reproduction 30 884-892. (doi:10.1038/srep16146)

Carlson AE, Westenbroek RE, Quill T, Ren D, Clapham DE, Hille B, Garbers DL \& Babcock DF 2003 CatSper1 required for evoked $\mathrm{Ca}^{2+}$ entry and control of flagellar function in sperm. Proceedings of the National Academy of Sciences 100 14864-14868. (doi:10.1073/ pnas.2536658100)

Chang H, Kim BJ, Kim YS, Suarez SS \& Wu M 2013 Different migration patterns of sea urchin and mouse sperm revealed by a microfluidic chemotaxis device. PloS ONE 8 e60587. (doi:10.1371/journal.pone.0060587)

Darszon A, Nishigaki T, Beltran C \& Treviño CL 2011 Calcium channels in the development, maturation, and function of spermatozoa. Physiological Reviews 91 1305-1355. (doi:10.1016/j.ydbio.2014.07.017)

Eisenbach M \& Giojalas LC 2006 Sperm guidance in mammals - an unpaved road to the egg. Nature Reviews. Molecular Cell Biology 7 276-285. (doi:10.1074/jbc.M115.690396)

Fisher HS \& Hoekstra HE 2010 Competition drives cooperation among closely related sperm of deer mice. Nature 463 801-803. (doi:10.1038/ nature08736)

Fukami K, Yoshida M, Inoue T, Kurokawa M, Fissore RA, Yoshida N, Mikoshiba K \& Takenawa T 2003 Phospholipase Cdelta4 is required for $\mathrm{Ca}^{2+}$ mobilization essential for acrosome reaction in sperm. Journal of Cell Biology 161 79-88. (doi:10.1083/jcb.200210057)

Gakamsky A, Armon L \& Eisenbach M 2009 Behavioral response of human spermatozoa to a concentration jump of chemoattractants or intracellular cyclic nucleotides. Human Reproduction 24 1152-1163. (doi:10.1093/humrep/den409)

Hyakutake T, Suzuki H \& Yamamoto S 2015 Effect of non-Newtonian fluid properties on bovine sperm motility. Journal of Biomechanics $\mathbf{4 8}$ 2941-2947. (doi:10.1016/j.jbiomech.2015.08.005)

Jansen RP 1978 Fallopian tube isthmic mucus and ovum transport. Science 201 349-351. (doi:10.1126/science.580814)

Jin $M$, Fujiwara E, Kakiuchi $Y$, Okabe $M$, Satouh $Y$, Baba SA, Chiba K \& Hirohashi N 2011 Most fertilizing mouse spermatozoa begin their acrosome reaction before contact with the zonapellucida during in vitro fertilization. Proceedings of the National Academy of Sciences $\mathbf{1 0 8}$ 4892-4896. (doi:10.1073/pnas.1018202108)

Lishko PV, Botchkina IL \& Kirichok Y 2011 Progesterone activates the principal $\mathrm{Ca}^{2+}$ channel of human sperm. Nature 471 387-391. (doi:10.1038/nature09767)

Lybaert P, Danguy A, Leleux F, Meuris S \& Lebrun P 2009 Improved methodology for the detection and quantification of the acrosome reaction in mouse spermatozoa. Histology and Histopathology 24 999-1007.

Macnab RM \& Koshland DE 1972 The gradient-sensing mechanism in bacterial chemotaxis. Proceedings of the National Academy of Sciences 69 2509-2512. (doi:10.1073/pnas.69.9.2509)

Miki K \& Clapham DE 2013 Rheotaxis guides mammalian sperm. Current Biology 23 443-452. (doi:10.1016/j.cub.2013.02.007)

Moore H, Dvoráková K, Jenkins N \& Breed W 2002 Exceptional sperm cooperation in the wood mouse. Nature 418 174-177. (doi:10.1038/ nature00832)

Mortimer ST 2000 CASA - practical aspects. Journal of Andrology 21 515-524.

Oren-Benaroya R, Orvieto R, Gakamsky A, Pinchasov M \& Eisenbach M 2008 The sperm chemoattractant secreted from human cumulus cells is progesterone. Human Reproduction 23 2339-2345. (doi:10.1093/ humrep/den265)

Pérez-Cerezales S, Boryshpolets S \& Eisenbach M 2015 Behavioral mechanisms of mammalian sperm guidance. Asian Journal of Andrology 17 628-632. (doi:10.1038/srep16146) 
Pietrobon EO, de los Angeles Monclus M, Alberdi AJ \& Fornés MW 2003 Progesterone receptor availability in mouse spermatozoa during epididymal transit and capacitation: ligand blot detection of progesterone-binding protein. Journal of Andrology 24 612-620. (doi:10.1002/j.1939-4640.2003.tb02713.x)

Ren D, Navarro B, Perez G, Jackson AC, Hsu S, Shi Q, Tilly JL \& Clapham DE 2001 A sperm ion channel required for sperm motility and male fertility. Nature 413 603-609. (doi:10.1038/35098027)

Smith DJ, Gaffney EA, Gadêlha H, Kapur N \& Kirkman-Brown JC 2009 Bend propagation in the flagella of migrating human sperm, and its modulation by viscosity. Cell Motility and the Cytoskeleton 66 220-236. (doi:10.1002/cm.v66:4)

Strünker T, Goodwin N, Brenker C, Kashikar ND, Weyand I, Seifert R \& Kaupp UB 2011 The CatSper channel mediates progesterone-induced $\mathrm{Ca}^{2+}$ influx in human sperm. Nature 471 382-386. (doi:10.1038/ nature09769)

Suarez SS 2008 Control of hyperactivation in sperm. Human Reproduction Update 14 647-657. (doi:10.1093/humupd/dmn029)

Suarez SS \& Dai X 1992 Hyperactivation enhances mouse sperm capacity for penetrating viscoelastic media. Biology of Reproduction 46 686-691. (doi:10.1007/s00441-015-2244-2)

Suarez SS \& Pacey AA 2006 Sperm transport in the female reproductive tract. Human Reproduction Update 12 23-37. (doi:10.1007/s00441015-2244-2)

Suarez SS, Katz DF, Owen DH, Andrew JB \& Powell RL 1991 Evidence for the function of hyperactivated motility in sperm.
Biology of Reproduction 44 375-381. (doi:10.1095/ biolreprod44.2.375)

Teves ME, Barbano F, Guidobaldi HA, Sanchez R, Miska W \& Giojalas LC 2006 Progesterone at the picomolar range is a chemoattractant for mammalian spermatozoa. Fertility and Sterility 86 745-749. (doi:10.1016/j.fertnstert.2006.02.080)

Uhler ML, Leung A, Chan SY \& Wang C 1992 Direct effects of progesterone and antiprogesterone on human sperm hyperactivated motility and acrosome reaction. Fertility and Sterility $\mathbf{5 8}$ 1191-1198.

Uñates DR, Guidobaldi HA, Gatica LV, Cubilla MA, Teves ME, Moreno A \& Giojalas LC 2014 Versatile action of picomolar gradients of progesterone on different sperm subpopulations. PloS ONE 9 e91181. (doi:10.1371/ journal.pone.0091181)

Xia J, Reigada D, Mitchell CH \& Ren D 2007 CATSPER channelmediated $\mathrm{Ca}^{2+}$ entry into mouse sperm triggers a tail-to-head propagation. Biology of Reproduction 77 551-559. (doi:10.1095/ biolreprod.107.061358)

Received 10 December 2015

First decision 8 January 2016

Revised manuscript received 31 January 2016

Accepted 22 February 2016 